\title{
AOR
}

Selected Papers of \#AolR2020:

The 22nd Annual Conference of the

Association of Internet Researchers

Virtual Event / 13-16 Oct 2021

\section{HOW ARE PUBLIC SPHERES TRANSNATIONAL? COMPARING NEWS AND SOCIAL MEDIA NETWORKS DURING THE MADRID CLIMATE TALKS}

\author{
Timothy Neff \\ University of Pennsylvania \\ Dariusz Jemielniak \\ Kozminski University
}

\section{Introduction}

In this study, we explore two parallel but related networks of discourse that arose during the United Nations Framework Convention on Climate Change (UNFCCC) negotiations of 2019 in Madrid: one produced by news media coverage of the talks; the other by Twitter users who shared news content about the talks. As these networks disseminated information about the UNFCCC's 25th Conference of the Parties (COP25), we see them as key channels of communication for a potentially transnational public sphere of dialogue and dissent, even if the existence or efficacy of such a transnational sphere is a matter of great debate (Fraser, 2007). Our aim is to compare the internal (within network) and external (across networks) homogeneity and heterogeneity of these two networks in terms of structure (e.g., language, geographic groups, etc.), dominant topics, and sentiments. We expect that moments of relative homogeneity will indicate how transnational public spheres may arise out of global news events.

\section{Theoretical framework}

We use public sphere theory (Habermas, 1989, 1998) and the concept of "thick big data analysis" (Jemielniak 2020) to understand how transnational public spheres might arise out of news media and Twitter networks. As public spheres are most often envisioned as requiring some degree of unity or consensus in order to influence policy action, and as climate change is in particular an issue requiring collective will and action to address, it is critical to know whether there is a realm of dialogue and dissent - a public sphere capable of influencing transnational climate policy making. Our use of "thick" analysis combines wide computational study with a qualitative focus on a selected sample in

Suggested Citation (APA): Neff, T., Jemielniak, D. (2021, October). How are Public Spheres Transnational? Comparing News and Social Media Networks During the Madrid Climate Talks. Paper presented at AolR 2021: The 22nd Annual Conference of the Association of Internet Researchers. Virtual Event: AolR. Retrieved from http://spir.aoir.org. 
order to provide nuanced understanding of network connections during COP25, a prominent example of a realm of transnational climate policy making, dialogue, and dissent that attracts international media attention.

\section{Data and methods}

We develop a multi-stage process to gather and analyze parallel network data from two sources: Twitter and MediaCloud. This process uses data produced by the act of linking to news content, either by media organizations or by Twitter users, to show affiliations between news organizations during COP25. These affiliations constitute the study's two media ecosystems. We gathered 138,501 tweets dated between 2019-12-02 00:00:00 and 2019-12-13 23:59:00, with the hashtag \#COP25. This timeframe roughly corresponds with COP25, which ran from Dec. 1, 2019, to Dec. 15, 2019, two days beyond the official end of the conference. We analyze the sentiment of these tweets and links to news media in a smaller subset of tweets. These links provide edges to build an affinity network showing how Twitter use produces relationships between news organizations. We also use Python module langdetect and the VADER Python script to understand language communities and sentiment in our corpus of tweets.

For our MediaCloud network of links created by professional media organizations, we take the top 50 news domains found in our Twitter data as a basis for creating a seed set of outlets for MediaCloud's "Topic Mapper" platform. This spidering process produces a directed network of links between news organizations and other web domains, showing relationships between these entities.

We then qualitatively assess the structure of these networks, their most prominent actors and topics, and, in the case of Twitter, the sentiment of tweets in order to assess the degree to which these networks demonstrate homogeneity and heterogeneity, both within each network (internally) and between them (externally).

\section{Findings}

Our Twitter network shows four communities strongly divided along language and national (geographic) lines: an English community with U.S. and U.K. news domains; three Spanish communities with Spanish and Chilean news domains; and a French community with French news domains. Strongly weighted edges between these communities are rare, but we find a focus on young climate activist Greta Thunberg that transcends these communities, thereby providing some homogeneity across the network. Twitter accounts related to certain news organizations also help amplify tweets to content from their own organizations. Analysis of all of the tweets in our corpus suggests different cultural practices, with German tweets more likely to generate replies and with the inclusion of news links in tweets somewhat suppressing Twitter interactions.

Our MediaCloud network, which mixes news and other sources due to the spidering process, includes 12 communities that also show strong division along language and geographic lines. Thunberg again is a dominant topic across many of these communities, but there also is some prominence for highly technical analysis of COP25, 
as well as the role of certain state and policy-making institutions, such as the UNFCCC. Media organizations under the same ownership tend to link to one another.

\section{Discussion}

Our findings shed light on how transnational public spheres might arise during international events such as COP25. First of all, the social capital of certain actors such as Greta Thunberg is capable of gathering attention and spurring discourse across diverse publics (external homogeneity). More research is required to understand the exact processes by which such actors accrue and spend such capital, though media attention is clearly a key source. Second, well-established international venues such as climate negotiations have the institutional infrastructure to sustain public spheres. Diverse publics seek to leverage the power of governments, media organizations, and policy-making bodies such as the UNFCCC to act on climate change, and therefore these institutions have prominent footprints in the transnational networks examined here. Third, media ownership is an important dimension of network structures, and research on network phenomena as diverse as the spread of misinformation and echo chambers should take account of such economic underpinnings to communication flows. Finally, cultural practices, including practices specific to the cultures of commercial institutions such as Twitter, can produce heterogeneity in transnational public spheres, which continue to show divisions along language, geographic, cultural lines.

In conclusion, our study shows that transnational public spheres can emerge out of relatively homogenous moments internal to networks and external to networks (i.e., across multiple networks). These moments arise at the intersection of media attention, cultural practices, and commercial and non-commercial (state) institutions, some of which have the capacity to effect changes in climate policy. At the same time, there are persistent divisions along language, geographic, and other lines that encourage the formation of distinct micro-spheres of networked actors (internal heterogeneity), as well as distinct social media practices (arguably cultures) that work to differentiate mass media networks from networks produced by a different set of publics on social media (external heterogeneity). These differences persist even during transnational events that draw widespread, sustained media focus around the globe. Moments of consensus and dissent will arise out of these contradictory currents of homogeneity and heterogeneity in transnational networks.

\section{References}

Fraser N (2007) Transnationalizing the public sphere: On the legitimacy and efficacy of public opinion in a post-Westphalian world. Theory, Culture \& Society, 24(4): 730.

Habermas J (1989) Structural Transformation of the Public Sphere, trans. Thomas Burger. MIT Press. 
Habermas J (1998) Between Facts and Norms: Contributions to a Discourse Theory of Law and Democracy. MIT Press.

Jemielniak, D (2020) Thick Big Data: Doing Digital Social Sciences. Oxford: Oxford University Press. 